\title{
Intestinal flora imbalance promotes alcohol-induced liver fibrosis by the TGF $\beta /$ smad signaling pathway in mice
}

\author{
DONG ZHANG ${ }^{1,2}$, XIUXIAN HAO $^{3}$, LILI XU $^{4}$, JING CUI $^{5}$, LI XUE $^{2}$ and ZIBIN TIAN ${ }^{6}$ \\ ${ }^{1}$ Medical College, Qingdao University; Departments of ${ }^{2}$ Gastroenterology and ${ }^{3}$ Endocrinology, Qingdao Center Hospital, \\ Qingdao, Shandong 266042; ${ }^{4}$ Department of Endocrinology, The Affiliated Hospital of Qingdao University, Qingdao, \\ Shandong 266003; ${ }^{5}$ Department of Emergency, The Third People's Hospital of Qingdao, Qingdao, Shandong 266041; \\ ${ }^{6}$ Department of Gastroenterology, The Affiliated Hospital of Qingdao University, Qingdao, Shandong 266003, P.R. China
}

Received August 26, 2015; Accepted April 21, 2017

DOI: $10.3892 / \mathrm{ol} .2017 .6762$

\begin{abstract}
Intestinal flora performs a crucial role in human health and its imbalance may cause numerous pathological changes. The liver can also affect the intestinal function through bile secretion via the enterohepatic cycle. The pathophysiological association between the gut and the liver is described as the gut-liver axis. The present study investigated the role of intestinal flora in alcohol-induced liver fibrosis. A total of 36 C57 mice were randomly and equally divided into 3 different dietary regimes: Group I (alcohol injury; received alcohol); group II (alcohol injury with flora imbalance; received alcohol plus lincomycin hydrochloride) and group III (alcohol injury with corrected flora imbalance; received alcohol, lincomycin hydrochloride and extra probiotics). The present study then investigated several indicators of liver damage. Alkaline phosphatase (ALP) levels, aspartate aminotransferase (AST) levels and alanine aminotransferase (ALT) levels in mice serum were studied. Masson staining and Annexin V-fluorescein isothiocyanate/propidium iodide double staining was also performed, and the expression of mothers against decapentaplegic homolog (smad) 3 and smad4 proteins in hepatic stellate cells (HSCs) of the mice was examined using western blot analysis. The levels of serum ALP, AST and ALT were the highest in group II mice, and all 3 levels decreased in group III mice compared with those from group II. The degree of liver fibrosis was aggravated in group II mice compared with group I mice. The apoptosis of HSCs was significantly inhibited in group II mice, but was increased in group III mice. The HSCs in group II mice exhibited higher expression of smad 3 and smad4, whilst group III mice (with corrected intestinal flora imbalance) exhibited downregulated expression of smad 3 and smad4. The present
\end{abstract}

Correspondence to: Mr. Zibin Tian, Department of Gastroenterology, The Affiliated Hospital of Qingdao University, 16 Jiangsu Road, Qingdao, Shandong 266003, P.R. China

E-mail: zibint98@163.com

Key words: intestinal flora imbalance, liver fibrosis, transforming growth factor $\beta / \mathrm{SMA} / \mathrm{MAD}$ homology signaling pathway data indicates that the intestinal flora perform a significant role in maintaining liver homeostasis. Furthermore, an imbalance of intestinal flora can exacerbate alcohol-induced liver fibrosis in mice through the transforming growth factor $\beta / \mathrm{SMA} / \mathrm{MAD}$ homology signaling pathway, which subsequently leads to more serious liver damage.

\section{Introduction}

Chronic alcoholic liver disease is typically caused by long-term excessive alcohol consumption, and includes alcoholic fatty liver, alcoholic hepatitis, alcoholic liver fibrosis and alcoholic cirrhosis. According to statistics, chronic alcoholic liver disease has become the leading cause of liver fibrosis and liver cancer in Europe and America (1). Liver fibrosis is the excessive accumulation and hyperplasia of extracellular matrix proteins resulting from chronic damage to the liver and characterized by abnormalities in the hepatic structure or the way it functions (2). It can be caused by autoimmune liver disease, drug-induced liver injury and chronic alcoholic liver disease. Previously, researchers have identified that transforming growth factor $\beta$ (TGF $\beta$ ) served an important role in the occurrence and development of liver fibrosis (3-5). Platelets in the liver cells can release large amounts of TGF $\beta$ following liver damage, which continuously promotes the synthesis and accumulation of extracellular matrix proteins, resulting in liver fibrosis. TGF $\beta$ can also enhance the activity of hepatic stellate cells (HSCs), which can in turn increase TGF $\beta$ synthesis and secretion, thus contributing to the development of liver fibrosis (6).

A complex community of microorganisms, termed intestinal flora, live in the digestive tract of the human body; $>500$ strains of intestinal flora exist in the human gut, the most common types being obligate anaerobes such as Bifidobacteria, E. coli, Enterococcus faecalis and Bacteroides (3). Intestinal flora performs a crucial role in human health. However, under certain abnormal conditions, bacterial translocation or a change of intestinal flora ratio can occur, resulting in various pathophysiological manifestations. The liver is connected to the intestinal flora system anatomically through the portal vein and mesenteric lymphatic system and continuously receives intestinal blood into the portal system. 
The liver also performs a defensive role in the detoxification of gut-derived toxins including lipopolysaccharides and microbial products depending on its innate immune system (7). Furthermore, the liver cannot only regulate metabolism and immune responses, but can also influence the intestinal function through bile secretion and the enterohepatic cycle. The pathophysiological association between the gut and the liver is described as the gut-liver axis. Over the past 10 years, understanding of the gut-liver axis has progressively increased, meaning that the impact of intestinal flora on the pathogenesis of chronic liver disease has received increased attention (8). It has been reported that patients with liver fibrosis often had abdominal distension, diarrhea and other gastrointestinal symptoms, but their symptoms alleviated following a period of treatment using probiotics, implying that their symptoms are associated with an intestinal flora imbalance (9). Therefore, it can be speculated that intestinal flora is associated with liver fibrosis.

The present study investigated the role of intestinal flora in the occurrence and development of liver fibrosis and explored its mechanism, providing a new theoretical basis for the treatment of liver fibrosis.

\section{Materials and methods}

Ethics statement. The animal experiments in the present study were approved by the committee on the Ethics of Qingdao University (Qingdao, China) in accordance with national and institutional policies. The animals received humane care and treatment in accordance with the Guide for the Care and Use of Laboratory Animals of Qingdao University.

Animals and treatments. Male C57B1/6 mice (6 weeks old; 30-35 g in weight) were bought from the laboratory animal center of The Academy of Military Medical Sciences (License number, SCXK Jing 2006-0009). A total of 36 mice were randomly allocated into 3 groups: Group I (alcohol injury group), group II (alcohol injury with flora imbalance group) and group III (alcohol injury with corrected flora imbalance group), using randomization software. The mice in group I were fed with lieber-deCarli liquid (Dyets, Inc., Bethlehem, PA, USA) diets containing $4 \%$ alcohol and $0.3 \%$ tetrachloromethane for 8 weeks to establish a mouse model with liver fibrosis. Subsequently, the mice were fed with lieber-deCarli liquid diets with equal energy of maltodextrin instead of alcohol for another 8 weeks. In addition to alcohol, the mice in group II were given lincomycin hydrochloride $(6 \mathrm{~g} / \mathrm{kg} / \mathrm{d})$ in the first 8 weeks and fed with the same diets to the ones in group I in the following 8 weeks. In group III, the mice received the same treatment to the ones in group II in the first 8 weeks and then were supplemented with $0.25 \mathrm{ml}$ probiotics (Lactobacillus casei subsp. rhamnosus strain, $480 \mathrm{mg} / \mathrm{ml}$ ) continuously for an additional 8 weeks. All mice were housed in cages by group at a temperature of $25^{\circ} \mathrm{C}$ and humidity of $60-70 \%$ with a8/16 h light/dark cycle (lights were turned on at 9:00 am and off at 5:00 pm).

To evaluate the degree of liver fibrosis and liver biochemical indicators in mice, half the mice in each group were sacrificed through cervical dislocation to harvest liver tissues and collect blood samples from the retro-orbital plexus; HSCs were additionally isolated from the remaining mice, as described below.
Pathologic assessments. The harvested liver tissues were fixed overnight in $10 \%$ formalin, embedded in paraffin, sectioned to a thickness of $4 \mu \mathrm{m}$ and stained with hematoxylin and eosin (H\&E). Following staining, histopathological characteristics of the liver tissues were examined under a light microscope. Masson staining (Shanghai Bo Valley Biological Technology Co., Ltd, Shanghai, China) was performed to evaluate the degree of liver fibrosis, according to the manufacturer's protocol. For histomorphometry, 3 midsagittal Masson's trichrome-stained sections were selected from each group. Images were captured using an optical microscope (ECLIPSE 50i; Nikon Corporation, Tokyo, Japan). The areas of blue (immature bone or collagen fibers) and red (remodeled or lamellar bone) staining were semiquantitatively determined using Image Pro Plus software (version 6.0; Media Cybernetics, Inc., Rockville, MD, USA). The area mean of total bone (red and blue) and remodeled bone staining was normalized to the control group and compared between the three groups. The hepatic pathologic assessments were qualitatively graded according to an alcohol induced disease (AID) histology score standard (10): i) Ballooning degeneration (0, none; 1 , mild to moderate; 2 , severe); ii) steatosis $(0, \leq 10 \% ; 1,>10-30 \% ; 2$, $>30-\leq 60 \% ; 3,>60 \%)$; iii) inflammation and necrosis $(0=$ none, $1=$ mild , $2=$ moderate, $3=$ severe $)$; iv) lobular fibrosis $(0=$ none, $1=$ mild, $2=$ moderate, $3=$ severe).

Biochemical analysis. The levels of alanine aminotransferase (ALT), aspartate aminotransferase (AST) and alkaline phosphatase (ALP) in the mice sera were detected with ALT, AST or ALP kits purchased from Jiancheng Technology Co., Ltd., (Nanjing, China).

HSC isolation. At 16 weeks, mice were anesthetized with $1 \%$ sodium pentobarbital. The mice livers were perfused to prepare for the digestion with D-Hanks (Beijing Solarbio Science \& Technology Co., Ltd., Beijing, China) via intubation of the portal vein until the livers turned white. Subsequently, mice livers were isolated from the site, placed on a dish and continuously perfused with $0.05 \%$ pronase and $0.025 \%$ collagenase (60 drops/min). Following the removal of the liver capsule and large blood vessels, the livers were made into a homogenate, filtered through 3 layers of gauze and centrifuged at $400 \mathrm{x} \mathrm{g}$ for $10 \mathrm{~min}$ at $25^{\circ} \mathrm{C}$. The supernatants were collected and then mixed with D-Hanks and DNase I until they were clear. Supernatants were carefully transferred onto the top of the cell separation solution and centrifuged at 1,400 $\mathrm{x} g$ for $20 \mathrm{~min}$ at $25^{\circ} \mathrm{C}$ to obtain HSCs. Following centrifugation HSCs were collected and washed 3 times with D-Hanks for western blot analysis. The purity of HSCs was identified by immunocytochemical staining of Desmin and $\alpha$ smooth muscle actin ( $\alpha$-SMA), as described previously (11).

Western blot analysis. Western blot analysis was performed in accordance with the standard protocols. Total protein extracts of HSCs were collected and lysed in ice-cold radioimmunoprecipitation assay buffer (Thermo Fisher Scientific, Inc., Waltham, MA, USA) with $10 \mu \mathrm{l} / \mathrm{ml}$ proteinase inhibitor cocktail (Thermo Fisher Scientific, Inc.) for $15 \mathrm{~min}$. The lysates were centrifuged at $12,000 \mathrm{x}$ g for $20 \mathrm{~min}$ at $4^{\circ} \mathrm{C}$. The protein concentration of each sample was determined 
by Coomassie brilliant blue staining. The cell lysates of each sample were mixed with 2X Laemmli buffer (Sigma-Aldrich; Merck KGaA, Darmstadt, Germany) and boiled for $5 \mathrm{~min}$. The proteins (50 $\mu \mathrm{g} /$ lane) were separated using $8 \%$ SDS-PAGE and transferred onto nitrocellulose membranes. The nitrocellulose blots were blocked with $5 \%$ skimmed milk for $1 \mathrm{~h}$, incubated at $25^{\circ} \mathrm{C}$ with primary antibodies against mothers against decapentaplegic homologs smad3 (cat. no., sc-101154) and smad4 (cat. no., sc-7966; both Santa Cruz Biotechnology Inc., Dallas, TX, USA), diluted with TBS/Tween-20 to 1:500, overnight at $4^{\circ} \mathrm{C}$. Subsequent to washing 3 times, the membranes were incubated for $40 \mathrm{~min}$ at $25^{\circ} \mathrm{C}$ with horseradish peroxidase conjugated rabbit anti-goat $\operatorname{IgG}$ (dilution, 1:1,000; cat. no., ab6721; Abcam, Cambridge, UK). The membranes were imaged using an enhanced-chemiluminescent kit (GE Healthcare, Chicago, IL, USA). Western blots were quantified using Image Pro Plus 5.1 (Media Cybernetics, Inc.), according to the kit protocol. All results were repeated 3 times.

Flow cytometry. Flow cytometry was utilized to measure the extent of apoptosis. Cells were stained with an annexin-V fluorescein isothiocyanate/propidium iodide kit (BD Biosciences, Franklin Lakes, NJ, USA) according to the manufacturer's protocol. The FlowSight instrument (BD Biosciences) was then used to perform flow cytometry and the data were analyzed using FlowJo software (version 7.6.1; Tree Star, Inc. Ashland, OR, USA).

Statistical analysis. All measured data were presented as the mean \pm standard deviation and analyzed with SPSS 18.0 software (SPSS Inc., Chicago, IL, USA). A one-way analysis of variance followed by a Fisher's least significant difference test and Kruskal-Wallis $\mathrm{H}$ test were used to assess differences among 3 groups according to the types of statistics. $\mathrm{P}<0.05$ was considered to indicate a statistically significant difference.

\section{Results}

ALT, ALP and AST levels in mice. The levels of ALT, ALP and AST in the mice of each group are shown in Fig. 1, which can demonstrate the degree of liver fibrosis to some extent. The mean values of AST, ALT and ALP in mice from the 3 groups were as follows: Group I, 54.87, 156.21 and 87.34 U/1, respectively; group II, 106.87, 234.21 and $139.34 \mathrm{U} / 1$, respectively; and group III, 76.87, 200.21 and 104.34 U/1, respectively. Mice in group II exhibited significantly higher serum ALT, ALP and AST levels $(\mathrm{P}<0.05)$ compared with those in group I and group III. Serum ALT, ALP and AST levels of the mice in group III were significantly lower compared with that of the mice in group II $(\mathrm{P}<0.05)$. These results indicate that intestinal flora imbalance can enhance the damage of alcohol to the liver.

Intestinal flora imbalance promoted alcohol induced liver fibrosis. Hepatic architecture was analyzed by H\&E staining and the degree of liver fibrosis was evaluated by Masson staining of liver tissue sections (Fig. 2). Although the mice in group I had normal hepatic lobule structure, inflammatory cell infiltration and hyperplasia of fibrous tissue existed around the portal area and central vein (Fig. 2A and B), suggesting that the lieber-deCarli liquid diets induced liver damage in mice. Compared with the
Table I. Alcohol induced disease histology score.

\begin{tabular}{lcccc}
\hline Group & $\begin{array}{c}\text { Hepatocyte } \\
\text { ballooning }\end{array}$ & $\begin{array}{c}\text { Liver } \\
\text { steatosis }\end{array}$ & $\begin{array}{c}\text { Liver } \\
\text { inflammation }\end{array}$ & Fibrosis \\
\hline III & $0.67 \pm 0.44$ & $0.81 \pm 0.68$ & $0.21 \pm 0.38$ & $0.29 \pm 0.49$ \\
II & $1.90 \pm 0.33$ & $2.48 \pm 0.58$ & $3.00 \pm 0.13$ & $2.79 \pm 0.29$ \\
I & $1.23 \pm 0.48$ & $2.13 \pm 0.48$ & $0.50 \pm 0.61$ & $0.53 \pm 0.55$ \\
\hline
\end{tabular}

Data are mean \pm standard deviation.

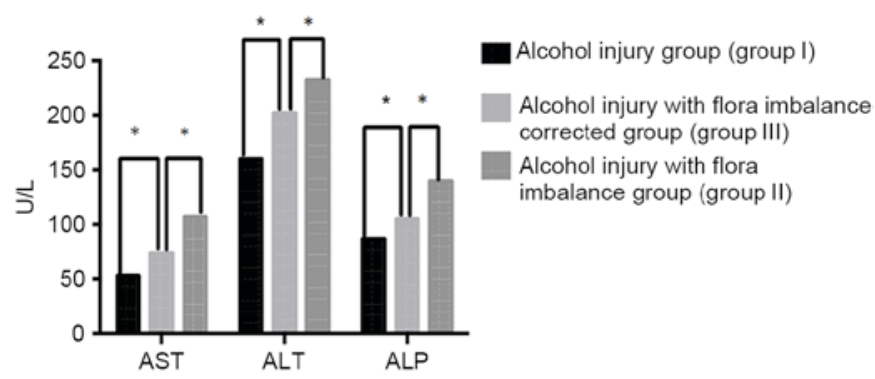

Figure 1. Serum AST, ALT and ALP levels in the mice among 3 groups. The mice in group II had the highest serum ALT, ALP and AST levels and the differences were statistically significant. Serum ALT, ALP and AST levels of the mice in group III were decreased compared with that of the mice in group II and the difference was statistically significant. " $\mathrm{P}<0.05$. AST, aspartate aminotransferase; ALT, alanine aminotransferase; ALP, alkaline phosphatase.

mice in group II, the structure of hepatic lobule of the mice in group III exhibited a greater degree of balloon degeneration, and decreased necrosis of the portal vein and surrounding liver cells. The collagen fibers in and around the lobules were also significantly reduced, and no fibrous septums were observed (Fig. 2C and D). Serious structural disorder of the hepatic lobule was observed in group II mice, along with significant hepatic inflammation including hepatocyte ballooning, degeneration and necrosis. The hyperplasia of liver collagen fibers was evident around the portal area and central vein, resulting in the formation of fibrous septums (Fig. 2E and F).

Intestinal flora imbalance induced hepatic pathological changes in mice in the 3 groups. The pathological assessments of livers were performed according to AID histology score standard. The scores of hepatocyte ballooning, liver steatosis, liver inflammation and fibrosis are summarized in Table I. Fig. 3 shows that the degree of liver damage was significantly higher in the mice of group II compared with the mice in group I. However, these effects were limited following the correction of intestinal flora imbalance in the mice of group III $(\mathrm{P}<0.05)$.

Intestinal flora imbalance inhibited apoptosis of HSCs in mice. HSCs perform an important role in producing collagen, which is an important process in development of liver fibrosis. To analyze the apoptosis of HSCs in the mice among 3 groups, Annexin V-FITC/PI double staining was performed. Apoptosis in HSCs was significantly inhibited in the livers of the mice in group II as compared with that in group I $(\mathrm{P}<0.05)$. 

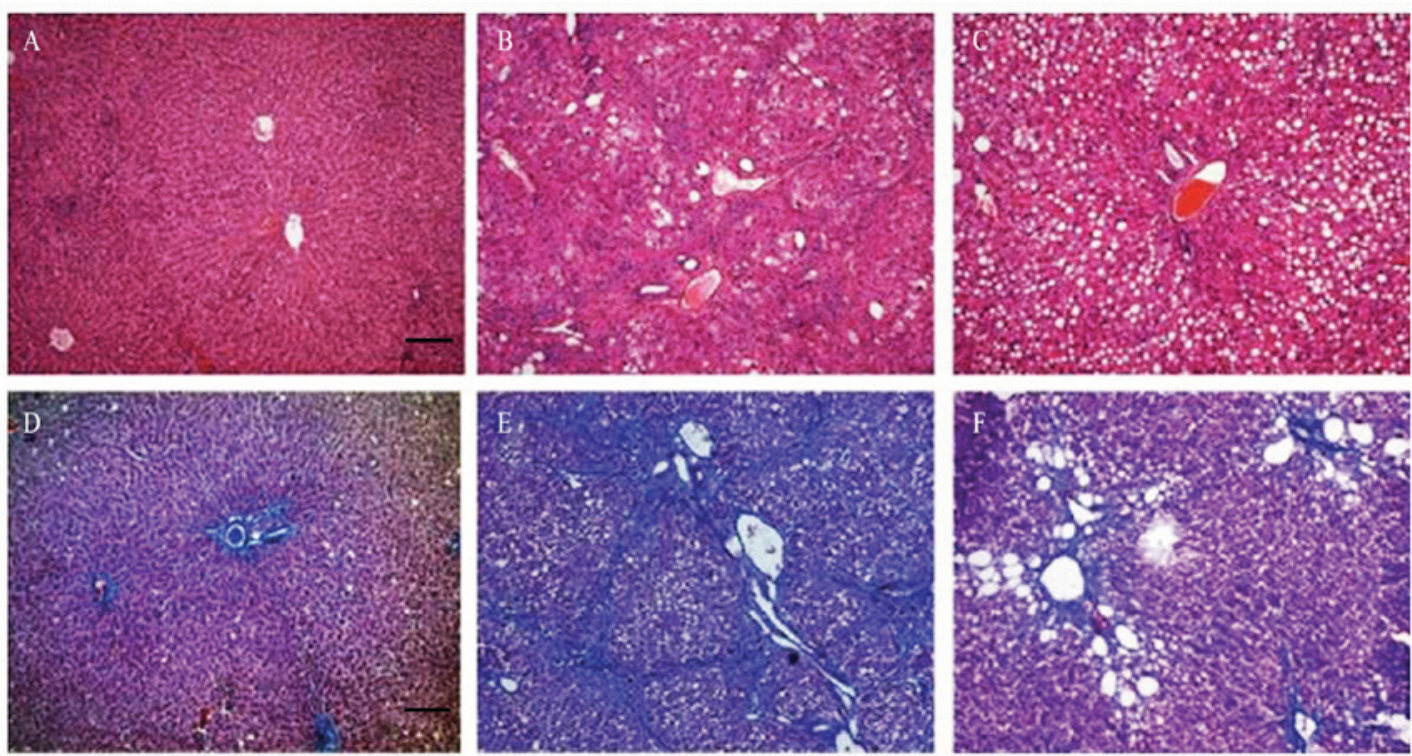

Figure 2. Evaluation of the severity of liver fibrosis. Sections of liver tissue from the (A) mice in group I, (B) group III and (C) group II were stained using hematoxylin and eosin. The degree of liver fibrosis was evaluated by Masson staining of liver tissue sections from the mice in (D) group I, (E) group III and (F) group II. Intestinal flora imbalance in the group II mice induced liver fibrosis. Scale bar, $50 \mu \mathrm{m}$.
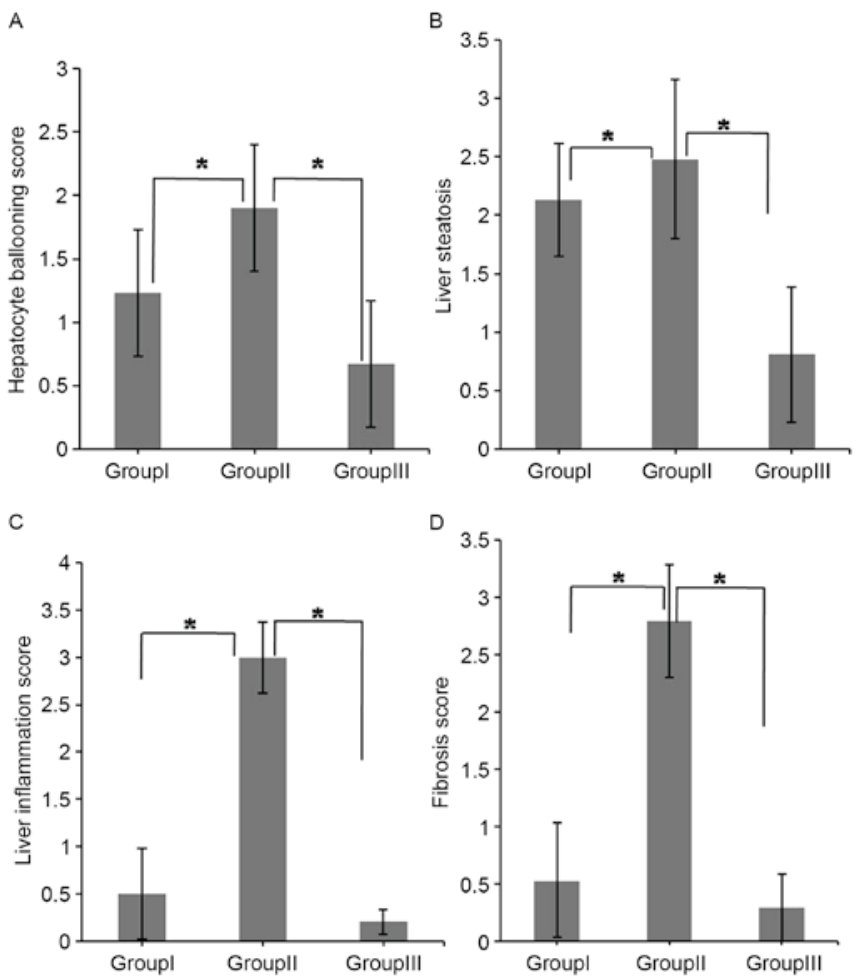

Figure 3. The hepatic pathologic scores according to alcohol induced disease histology score standard: (A) Hepatocyte ballooning; (B) liver steatosis score; (C) liver inflammation score; and (D) fibrosis score. The pathological changes in the liver of group II mice significantly increased compared with the mice in group I, whereas these pathological changes were significantly rescued in group III mice compared with group II mice. Error bars represent standard deviation. ${ }^{*} \mathrm{P}<0.05$.

The present study also observed that correcting the intestinal flora imbalance in the mice of group III significantly increased apoptosis in HSCs compared with that in group I $(\mathrm{P}<0.05$; Fig. 4). These data indicate that an intestinal flora imbalance may be associated with the proliferation and activation of HSCs.

Association of intestinal flora imbalance and TGF $\beta /$ smad signaling pathway. To investigate whether the TGF $\beta / \mathrm{smad}$ signaling pathway is involved in the role of intestinal flora imbalance in alcohol-induced liver fibrosis, western blot analysis was performed to detect the expression levels of smad 3 and smad4 in HSCs among 3 groups. The present data shows that the expression levels of $\operatorname{smad} 3$ and $\operatorname{smad} 4$ were significantly upregulated in HSCs of the mice in group II compared with that in group I ( $\mathrm{P}<0.01$; Fig. 5A). By contrast, the expression levels of smad3 and smad4 were significantly downregulated in HSCs of the mice in group III compared to that in the mice of group II $(\mathrm{P}<0.01 ;$ Fig. 5B).

\section{Discussion}

Several studies have indicated that there are associations between intestinal flora imbalance and liver injury (12-14). The present study investigated the association between intestinal flora imbalance and liver fibrosis in 4 ways: Analysis of serum AST, ALT and ALP levels; observing the hepatic pathological changes; monitoring the proliferation rate of HSCs; and monitoring the expression of smad3 and smad4.

It has previously been reported that the levels of serum ALT, AST and ALP were positively correlated with liver injury and fibrosis and used as indices of accessory diagnosis (15-17). The present study found that the levels of serum AST, ALT and ALP in mice of the alcoholic injury with intestinal flora imbalance group (group II) were significantly increased compared with that in the alcoholic injury group (group I) $(\mathrm{P}<0.05)$; while these diagnosis indices in mice of the alcoholic injury with corrected intestinal flora group (group III) were significantly decreased compared with that in group II $(\mathrm{P}<0.05)$, which suggested that the reduction of intestinal 

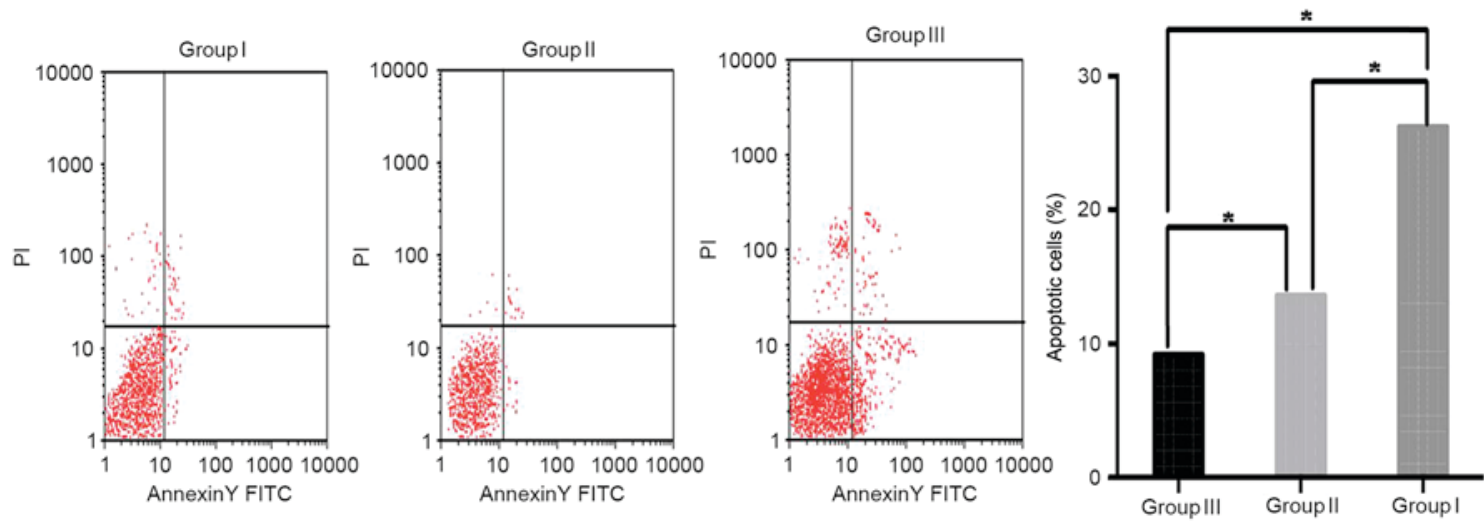

Figure 4. Apoptosis in HSCs of the mice among 3 groups was examined using Annexin-V fluorescein isothiocyanate/propidium iodide double staining and analyzed using flow cytometry. Apoptosis in HSCs was significantly inhibited in the livers of group II mice as compared with group I mice. Correcting the intestinal flora imbalance in the group III mice significantly increased apoptosis in HSCs compared with that in group I. "P<0.05. HSC, hepatic stellate cell.
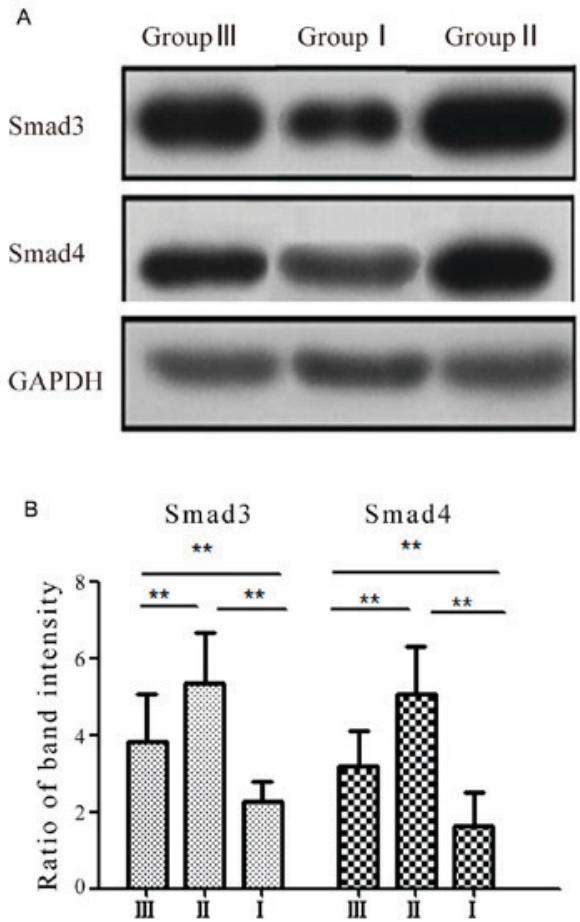

Figure 5. Expression of smad3 and 4 in mouse HSCs. (A) The expression levels of smad 3 and smad4 in HSCs of the mice among 3 groups were detected by western blot analysis. (B) Quantification of the western blot analysis. The expression levels of smad 3 and 4 were significantly upregulated in the HSCs of the mice in group II compared with group III. Error bars represent standard deviation. ${ }^{* *} \mathrm{P}<0.01$. HSC, hepatic stellate cell.

flora contributed to increased vulnerability to alcohol induced liver fibrosis. Furthermore, in order to investigate the role of intestinal flora imbalance in liver fibrosis, the present study performed H\&E staining and Masson staining of liver tissue sections among 3 groups and found a relatively high degree of liver fibrosis in the mice of group II compared with that in the mice of group I and group III, primarily manifested increased hepatocyte ballooning degeneration and necrosis, inflammatory cell infiltration, and a significantly extended area of collagen fiber. Liver fibrosis may be caused by impaired intestinal barrier function following an imbalance in intestinal flora. When the intestinal barrier is damaged, harmful substances such as bacteria and bacterial metabolites in the intestine can invade the liver through the enterohepatic cycle. This leads to over-activation of the immune system and adverse immune reactions, which subsequently cause vast inflammatory cell infiltration, hepatocyte degeneration and necrosis (15).

A HCS is a type of mesenchymal cell in the liver that has characteristics of muscle, adipose and fibroblast cells. HSCs regulate blood flow and liver fibrosis, and help to maintain a metabolic balance of Vitamin A $(17,18)$. In physiological conditions, HSCs grow slowly and are almost dormant, producing little collagen. However, when pathological changes such as alcoholic induced liver damage occur in the liver, liver cells secrete numerous cytokines, including tumor necrosis factor- $\alpha$ (TNF- $\alpha$ ) and TGF $\beta$, which promote HSCs to lose lipids and undergo morphological changes into fibroblasts. Liver cells release excess extracellular matrixes (ECMs), causing the proliferation and activation of HSCs (19). Extensive ECM accumulation in the sinusoids and perisinusoidal space is one of pathological features of liver fibrosis, and so the proliferation and activation of HSCs are cytological elements causing liver fibrosis (20). The present data show that apoptosis of HSCs in group II mice was significantly inhibited compared with that of group I mice, meaning that HSCs are more susceptible to proliferate and differentiate into fibroblasts. Conversely, apoptosis of HSCs in group III mice increased significantly compared with that of group II mice, suggesting that liver fibrosis may be recovered following the correction of the intestinal flora imbalance.

With the depth of research on pathological fibrosis, an increasing number of studies consider that smad 3 and smad4 are critical in the development of pathological fibrosis. Huang et al (21) identified that the expression of smad3 in patients with renal fibrosis was increased compared with that in controls. A study by Schwartze et al (22) found that the expression of smad3 and smad4 was significantly upregulated in patients with pulmonary fibrosis. Additionally, Zhu et al (23) reported that the degree of liver fibrosis was alleviated following inhibiting the expression of $\operatorname{smad} 3$ in rats. Therefore, smad3 may perform an important role in promoting pathological fibrosis in different organs. There is increasing evidence that smad 3 and smad4 serve as mediators in the TGF- $\beta$ signaling pathway and perform important roles in the occurrence of liver fibrosis (24). TGF- $\beta$ is a major cell-signaling pathway involved in activating 
HSCs and increasing the synthesis of collagen and other extracellular matrix proteins by regulating downstream pathways such as smad proteins. The present study detected the expression of smad 3 and smad 4 in HSCs of mice among 3 groups and identified that significantly increased expression levels of smad3 and smad4 were observed in group II mice compared with the mice in group I. Additionally, the expression levels of smad 3 and smad4 significantly reduced in HSCs of group III mice compared with those observed in group II mice. The present data indicated that the TGF $\beta /$ smad signaling pathway is involved in the effect of intestinal flora imbalance on liver fibrosis.

A previous study (24) identified that intestinal flora imbalance can increase the level of bacterial endotoxin, and subsequently upregulate the secretion of inflammatory factors such as TNF- $\alpha$, interleukin (IL)-1 $\beta$ and IL-6. These inflammatory factors enhanced oxidative stress in the liver, which led to liver fibrosis and lipid peroxidation. The inflammatory factors also activated TGF $\beta$ that exacerbates liver fibrosis. It is known that TGF $\beta$ can transduce proteins by regulating downstream signaling factors such as smad family proteins $(25,26)$. Therefore, intestinal flora imbalance increases alcohol induced liver fibrosis in mice by upregulating the TGF $\beta /$ smad signaling pathway, which is shown by the downregulation of smad3 and smad4 in HSCs of the group III mice.

In summary, liver fibrosis and intestinal flora imbalance influence each other, leading to a cycle in which liver fibrosis produces intestinal flora imbalance, which in turn aggravates liver fibrosis. The present study demonstrated that correcting intestinal flora imbalance is necessary to break this cycle and maintain liver homeostasis. Subsequent to correcting intestinal flora imbalance, the proliferation and activation of HSCs was attenuated, which reduced excessive production of extracellular matrixes by downregulating the TGF $\beta /$ smad signaling pathway. Therefore, correcting intestinal flora imbalance is crucial and can be an effective method in the treatment of liver fibrosis.

\section{Acknowledgements}

The present study was supported by the Medical College of Qingdao University (Qingdao, China) and the Department of Gastroenterology in Qingdao Center Hospital (Qingdao, China).

\section{References}

1. Blachier M, Leleu H, Peck-Radosavljevic M, Valla DC and Roudot-Thoraval F: The burden of liver disease in Europe: A review of available epidemiological data. J Hepatol 58: 593-608, 2013.

2. Bataller R and Brenner DA: Liver fibrosis. J Clin Invest 115: 209-218, 2005.

3. Szabo G and Bala S: Alcoholic liver disease and the gut-liver axis. World J Gastroenterol 16: 1321-1329, 2010.

4. Wu ZW, Ling ZX, Lu HF, Zuo J, Sheng JF, Zheng SS and Li LJ: Changes of gut bacteria and immune parameters in liver transplant recipients. Hepatobiliary Pancreat Dis Int 11: 40-50, 2012.

5. Yang Y, Kim B, Park YK, Koo SI and Lee JY: Astaxanthin prevents TGF $\beta 1$-induced pro-fibrogenic gene expression by inhibiting Smad3 activation in hepatic stellate cells. Biochim Biophys Acta 1850: 178-185, 2015.

6. Liu Q, Duan ZP, Ha DK, Bengmark S, Kurtovic J and Riordan SM: Synbiotic modulation of gut flora: Effect on minimal hepatic encephalopathy in patients with cirrhosis. Hepatology 39: $1441-1449,2004$
7. Lata J, Novotný I, Príbramská V, Juránková J, Fric P, Kroupa R and Stibůrek O: The effect of probiotics on gut flora, level of endotoxin and child-pugh score in cirrhotic patients: Results of a double-blind randomized study. Eur J Gastroenterol Hepatol 19: 1111-1113, 2007.

8. Shukla S, Shukla A, Mehboob S and Guha S: Meta-analysis: The effects of gut flora modulation using prebiotics, probiotics and synbiotics on minimal hepatic encephalopathy. Aliment Pharmacol Ther 33: 662-671, 2011.

9. Bauer TM, Schwacha H, Steinbrückner B, Brinkmann FE, Ditzen AK, Aponte JJ, Pelz K, Berger D, Kist M and Blum HE: Small intestinal bacterial overgrowth in human cirrhosis is associated with systemic endotoxemia. Am J Gastroenterol 97: 2364-2370, 2002.

10. Maddrey WC: Alcohol-induced liver disease. Clin Liver Dis 4: 115-131, vii, 2000.

11. Yan G, Li B, Xin X, Xu M, Ji G and Yu H: MicroRNA-34a promotes hepatic stellate cell activation via targeting ACSL1. Med Sci Monit 21: 3008-3015, 2015

12. Jarzembowski T, Daca A, Bryl E, Wiśniewska K, Gołębiewska J, Dębska-Ślizień A, Rutkowski B and Witkowski J: Increased pheromone cCF10 expression in Enterococcus faecalis biofilm formed by isolates from renal transplant patients. Curr Microbiol 65: 656-659, 2012.

13. Li L, Wu Z, Ma W, Yu Y and Chen Y: Changes in intestinal microflora in patients with chronic severe hepatitis. Chin Med J (Engl) 114: 869-872, 2001.

14. Schreiber S, Rosenstiel P, Albrecht M, Hampe J and Krawczak M: Genetics of Crohn disease, an archetypal inflammatory barrier disease. Nat Rev Genet 6: 376-388, 2005.

15. Housset $\mathrm{C}$ and Guéchot J: Hepatic fibrosis: Physiopathology and biological diagnosis. Pathol Biol (Paris) 47: 886-894, 1999 (In French).

16. Liao SL, Kao TK, Chen WY, Lin YS, Chen SY, Raung SL, Wu CW, Lu HC and Chen CJ: Tetramethylpyrazine reduces ischemic brain injury in rats. Neurosci Lett 372: 40-45, 2004.

17. Nozaki Y, Fujita K, Wada K, Yoneda M, Kessoku T, Shinohara Y, Imajo K, Ogawa Y, Nakamuta M, Saito S, et al: Deficiency of iNOS-derived NO accelerates lipid accumulation-independent liver fibrosis in non-alcoholic steatohepatitis mouse model. BMC Gastroenterol 15: 42, 2015.

18. Reyes-Gordillo K, Shah R, Arellanes-Robledo J, Hernández-Nazara Z, Rincón-Sánchez AR, Inagaki Y, Rojkind M and Lakshman MR: Mechanisms of action of acetaldehyde in the up-regulation of the human $\alpha 2$ (I) collagen gene in hepatic stellate cells: Key roles of Ski, SMAD3, SMAD4, and SMAD7. Am J Pathol 184: 1458-1467, 2014.

19. O'Reilly S, Ciechomska M, Cant R and van Laar JM: Interleukin-6 (IL-6) trans signaling drives a STAT3-dependent pathway that leads to hyperactive transforming growth factor- $\beta$ (TGF- $\beta$ ) signaling promoting SMAD3 activation and fibrosis via Gremlin protein. J Biol Chem 289: 9952-9960, 2014.

20. Sun YB, Qu X, Li X, Nikolic-Paterson DJ and Li J: Endothelial dysfunction exacerbates renal interstitial fibrosis through enhancing fibroblast Smad3 linker phosphorylation in the mouse obstructed kidney. PLoS One 8: e84063, 2013.

21. Huang XZ, Wen D, Zhang M, Xie Q, Ma L, Guan Y, Ren Y, Chen J and Hao CM: Sirtl activation ameliorates renal fibrosis by inhibiting the TGF- $\beta /$ Smad3 pathway. J Cell Biochem 115: 996-1005, 2014.

22. Schwartze JT, Becker S, Sakkas E, Wujak ŁA, Niess G, Usemann J, Reichenberger F, Herold S, Vadász I, Mayer K, et al: Glucocorticoids recruit Tgfbr 3 and Smad1 to shift transforming growth factor- $\beta$ signaling from the Tgfbrl/Smad2/3 axis to the Acvrl1/Smad 1 axis in lung fibroblasts. J Biol Chem 289: 3262-3275, 2014.

23. Zhu JN, Chen R, Fu YH, Lin QX, Huang S, Guo LL, Zhang MZ, Deng CY, Zou X, Zhong SL, et al: Smad3 inactivation and MiR-29b upregulation mediate the effect of carvedilol on attenuating the acute myocardium infarction-induced myocardial fibrosis in rat. PLoS One 8: e75557, 2013.

24. Lv KY, Zhong QS, Liu XF, Zhu SH, Xiao SC, Wang GY, Ma B and Xia ZF: Deficiency of Smad3 results in enhanced inducible nitric oxide synthase-mediated hypotension in lipopolysaccharide-induced endotoxemia. J Surg Res 187: 640-645, 2014.

25. Ji F, Fu SJ, Shen SL, Zhang LJ, Cao QH, Li SQ, Peng BG, Liang LJ and Hua YP: The prognostic value of combined TGF- $\beta 1$ and ELF in hepatocellular carcinoma. BMC Cancer 15: 116, 2015.

26. Kamato D, Burch ML, Piva TJ, Rezaei HB, Rostam MA, Xu S, Zheng W, Little PJ and Osman N: Transforming growth factor- $\beta$ signalling: Role and consequences of Smad linker region phosphorylation. Cell Signal 25: 2017-2024, 2013. 